actions themselves. Consequently, these reaction-diffusion systems can show selforganizing ability.

Interactions between knirps and its anterior neighbour, Krüppel, have already been described ${ }^{19}$. It will be interesting to see how the posterior gap genes regulate one another and if it is possible to explain the formation of the posterior pattern as a consequence of these interactions.

Two sets of observations are consistent with this sort of mechanism. First, the posterior system has self-organizing properties which contrast with the anterior bicoid system $^{4}$. Most dramatically, nanos factor injected into the middle of an embryo which lacks both bicoid and nanos induces abdominal segments radiating from the poles of the embryo, not the site of injection (bicoid activity induces anterior segments radiating from the site of injection). This difference may be due to the properties of the zygotic genes involved in posterior patterning ${ }^{4}$.

Second, the posterior pattern can respond to changes in zygotic gap gene activity. For example, Lehmann has shown that pumilio mutants, which prevent diffusion of the nanos activity, can be suppressed by mutants in the terminal zygotic gap gene tailless ${ }^{2)}$.

The present work raises many questions, above all, what determines position in the posterior region? Is there an undetected maternal gradient? Do gap-gene interactions spontaneously generate the pattern, or is something else going on?

Ken Howard is in the Center for Neurobiology and Behavior, Howard Hughes Medical Institute, Columbia University, New York, New York 10032, USA.

\title{
METEOROLOGY
}

\section{It never rains, but it pours}

\section{Ian N. James}

Superficially, it can seem that the character of the weather persists in one mode for considerable periods before switching to some very different mode. For example, the early part of this winter was remarkable dry, warm and settled in western Europe. Since mid-February, an equally persistent unsettled regime associated with a sequence of vigorous depressions has dominated the weather (compare the adage in the title). Such

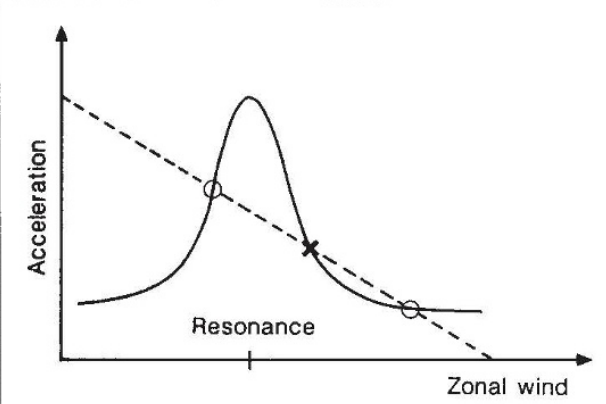

stable; $X$ unstable) if this wave drag balances the relaxation to the undisturbed state; the existence of multiple equilibria depends upon the sharpness of the resonance shown in the wave-drag curve. The introduction of weak nonlinearity can 'bend' the resonance peak, so that multiple equilibria with the same zonal wind are possible ${ }^{6}$.

superficial impressions are borne out by more careful analysis of atmospheric data. There is evidence that the amplitude of planetary-scale 'Rossby' waves in the Northern Hemisphere can switch from a larger to a smaller amplitude mode and back $^{1}$. Hansen and Sutera ${ }^{2}$ now suggest in the December issue of the Journal of Atmospheric Sciences that similar bimodality is present in the Southern Hemisphere. Understanding this result is important, because theories of bimodality rely heavily on the generation of planetary waves as the mid-latitude westerlies rise over mountain chains. There is much less scope for such forcing in the Southern Hemisphere, suggesting that present theories of bimodality may be inadequate.

Hansen and Sutera examined a series of daily 500-millibar geopotential height fields, analysed by the European Centre for Medium Range Forecasts, for a period of 4.5 years over the Southern Hemisphere. The greatest amplitude is associated with zonal wavenumber 3 (that is,

Bimodality can occur when there are two equilibrium states in the weather system. The figure shows how multiple equilibria arise in the simplest model ${ }^{5}$. It is supposed that the flow tends to relax to some undisturbed state in the absence of any mountains (broken line). In the presence of mountains there is an extra drag (solid curve) which depends upon the amplitude of the excited Rossby waves (low-frequency transverse waves which dominate the planetary-scale response to thermal and orographic forcing). Equilibria exist $(O$,

three maxima and minima around the latitude circle) in the middle latitudes. The authors calculated the probability density of different wavenumber 3 amplitudes after removing any long-term trends. For the winter, there was a clearly bimodal distribution, whereas there was only a single maximum in the summer. Examination of the time series shows that each amplitude phase could persist for periods of 20-30 days. For other times, the authors note a more irregular fluctuation of amplitude. Combining the fields for periods in which the large-amplitude or low-amplitude wavenumber 3 was present reveals a clear switching from zonal wavenumber 1 to zonal wavenumber 3 .

Such bimodality in atmospheric data seems to be a hemispheric rather than a local phenomenon. Theories have been developed (see box) which predict bimodality using simple models. The crucial ingredients in such models are: first, a source of planetary-scale Rossby-wave activity in the middle latitudes, generally provided by flow over large-scale mountain ranges; and second, a resonance in the response of the large-scale flow to such forcing.

In the Southern Hemisphere, it is not clear that mountain topography can generate the observed wavenumber 3 (ref. 3). The Andes, the only considerable mountain range of the southern midlatitudes, force a response which is confined to the subtropics. An alternative source of wave activity, perhaps associated with variations of sea surface temperatures with longitude, must be sought. A more serious problem is the need for resonance. This can occur if the zonal flow acts as a waveguide, confining the wave activity to the mid-latitudes. A wave train radiated from the source will eventually propagate back into the source region. If the waves have the right phase, resonance occurs. In the simple theories, a guide is simply provided by confining the flow with rigid boundaries to north and south; these reflect Rossby waves. But no such reflecting boundaries exist in the real atmosphere. Indeed a fundamental property of the global circulation is the largely one-way dispersion of Rossby waves from mid-latitudes into the tropics.

It is intriguing that a weak waveguide behaviour for just wavenumber 3 might be possible between $50^{\circ} \mathrm{S}$ and $70^{\circ} \mathrm{S}$ (refs 3 and 4). But such a waveguide would be extremely leaky and unlikely to generate the sharp resonance needed to explain the observed bimodality. For us to understand these observations more satisfactorily, it is necessary to develop a theory of multiple equilibria which does not depend on the presence of reflecting boundaries to the flow domain. In a system as highly nonlinear as the atmosphere, it would be no surprise to find that multiple equilibria are possible. The challenge is to find a specific mechanism.

Ian N. James is in the Department of Meteorology, Reading University, Reading RG6 2AU, UK.

1. Hansen, A. R. \& Sutera. A. J. atmos. Sci. 43, 3250-3265 (1986).

2. Hansen, A. R. \& Sutera, A. J. atmos Sci. 45, 3771-3783 (1988).

3. James, I. N. Q. J/R. met. Soc. 114, 619-637 (1988).

4. Held. I. M. in Large Scale Dynamical Processes in the Atmosphere (eds Hoskins, B. J. \& Pearce, R. P.) 127-168 (Academic, London, 1983)

5. Charney, J. G. \& DeVore, J. G. J. atmos. Sci. 36, 12051216 (1979).

6. Benzi, R., lalori, G. \& Sutera, A. J. atmos. Sci, 45, 3313 3319 (1988). 